\title{
Fulminant Haemolysis Following Endoscopic Retrograde Cholangiopancreatography
}

\author{
Mário Bibi ${ }^{1}$, Susana Viana ${ }^{1}$, Cátia Leitão², Rui Moço ${ }^{1}$, Yuliana O. Eremina ${ }^{3,4}$ \\ ${ }^{1}$ Internal Medicine Department, Hospital Pedro Hispano, Porto, Portugal \\ ${ }^{2}$ Gastroenterology Department, Hospital Pedro Hispano, Porto, Portugal \\ ${ }^{3}$ Clinical Pathology Department, Hospital Pedro Hispano, Porto, Portugal \\ ${ }^{4}$ EPIUnit, Instituto de Saúde Pública, Universidade do Porto, Porto, Portugal
}

Received: 31/07/2021

Accepted: $13 / 08 / 2021$

Published: 01/09/2021

How to cite this article: Bibi M, Viana S, Leitão C, Moço R, Eremina YO, Fulminant haemolysis following endoscopic retrograde cholangiopancreatography. EJCRIM 2021;8: doi:10.12890/2021_002811.

Conflicts of Interests: The authors declare there are no competing interests.

This article is licensed under a Commons Attribution Non-Commercial 4.0 License

\section{ABSTRACT}

We report the case of a 77-year-old-man with a history of type 2 diabetes mellitus who underwent endoscopic retrograde cholangiopancreatography (ERCP) because of a gallstone in the common bile duct. Thirty-six hours after the procedure, the patient developed persistent fever and epigastric pain associated with de novo jaundice. Massive haemolysis (with exuberant spherocytosis) occurred and patient died in 3 hours. Clostridium perfringens was isolated in the blood cultures. Massive haemolysis associated with C. perfringens has a high mortality rate. Management involves a high index of suspicion after gastrointestinal procedures like ERCP, surgical consultation, antibiotic therapy, transfusion of red cell concentrates and, potentially, hyperbaric oxygen therapy.

\section{LEARNING POINTS}

- Endoscopic retrograde cholangiopancreatography (ERCP) can be complicated by Clostridium perfringens bacteraemia with devastating consequences.

- C. perfringens infection should be suspected in an icteric, febrile patient with abdominal pain, especially if intravascular haemolysis is present.

- Management of intravascular haemolysis and inflammation in a patient following ERCP should be multidisciplinary, involving surgery when needed and potentially hyperbaric oxygen therapy; penicillin or penicillin-derived antibiotics associated with clindamycin or metronidazole are the mainstays of antibiotic therapy.

\section{KEYWORDS}

Clostridium perfringens, haemolysis, spherocytosis, endoscopic retrograde cholangiopancreatography, sepsis

\section{CASE DESCRIPTION}

A 77-year-old man with a history of type 2 diabetes mellitus was brought to our emergency department because of polydipsia and polyuria for the previous 2 months. He had decompensated diabetes mellitus, with a haemoglobin A1c of 12.2\%, but did not meet the criteria for a hyperglycaemic hyperosmolar state or diabetic ketoacidosis. Investigation was remarkable for an elevated gamma-glutamyltransferase (684 U/l; normal $(\mathrm{N})<64)$, alkaline phosphatase (472 U/I; $\mathrm{N}<150)$, aspartate transaminase (74 $\mathrm{U} / \mathrm{l} ; \mathrm{N}<34)$ and alanine transaminase $(102$ $\mathrm{U} / \mathrm{I} ; \mathrm{N}<55)$, with normal total bilirubin. Initial blood cultures were negative. An initial abdominal CT scan showed multiple pancreatic cystic lesions, judged to be benign with endoscopic ultrasound, which, however, identified a gallstone in the common bile duct (image not available). 


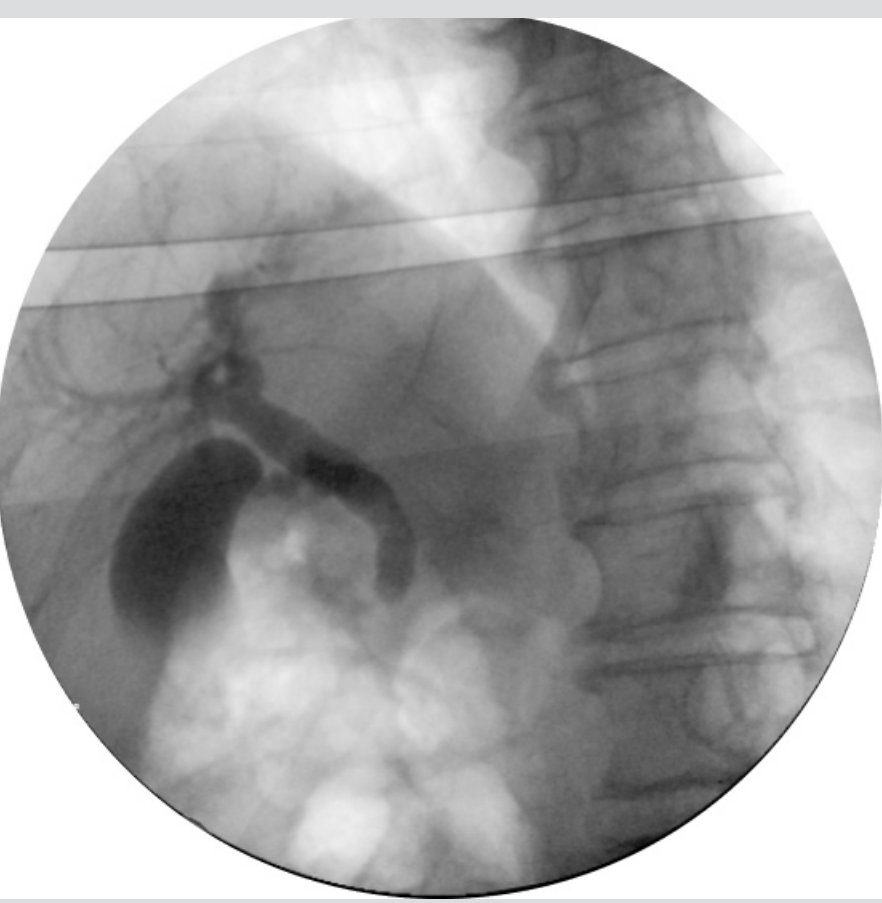

Figure 1. Endoscopic retrograde cholangiopancreatography showing an occlusion cholangiogram without evidence of gallstones

Following this, endoscopic retrograde cholangiopancreatography (ERCP) was performed and only found biliary sludge (Fig. 1).

The patient did not receive pre-procedure antibiotics. Twelve hours after the procedure, the patient was asymptomatic but the biochemistry showed de novo elevations of total bilirubin ( $3.3 \mathrm{mg} / \mathrm{dl}, \mathrm{N}<1.2$, with predominant direct bilirubin fraction), C-reactive protein (102 mg/l, $\mathrm{N}<5$ ) and leucocyte count $\left(17.56 \times 10^{3} / \mu \mathrm{l}\right)$, with normal pancreatic amylase $(36 \mathrm{U} / \mathrm{l}, \mathrm{N}<53)$. After 24 hours, the patient was icteric and developed a persistent fever and epigastric pain. Despite the initiation of piperacillin-tazobactam, the patient rapidly evolved to cardiorespiratory collapse and death in 3 hours. The clinical conditions did not allow a CT scan to be carried out. Blood biochemical analysis was not possible as there was no clot formation and no serum separation after centrifugation. The complete blood count showed an acute reduction in haemoglobin concentration (from $12.8 \mathrm{~g} / \mathrm{dl}$ to $9.8 \mathrm{~g} / \mathrm{dl}$ in 24 hours), with a mean corpuscular haemoglobin increase from $31 \mathrm{pg}$ to 35.3 pg, reticulocytosis of $11 \%$ and increasing leucocytosis $\left(39.83 \times 10^{3} / \mu \mathrm{l}\right)$ with neutrophil left shift. Haematocrit also abruptly reduced (from $37 \%$ to $27 \%$ and during cardiopulmonary resuscitation to less than $15 \%)$. The blood smear showed exuberant spherocytosis and other alterations typical for haemolysis and leucocyte alterations typical for sepsis (Fig. 2).

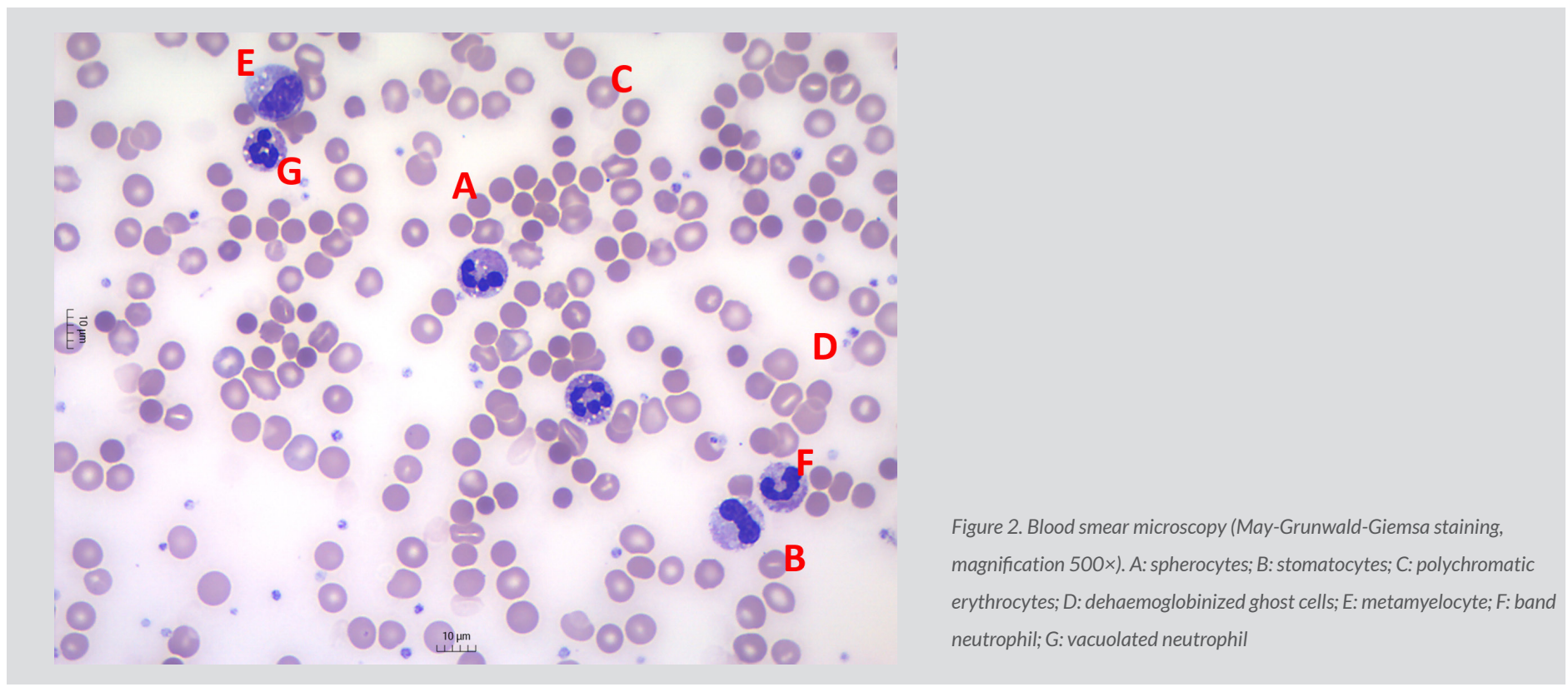


Clostridium perfringens was isolated in the blood cultures. An autopsy was not performed.

\section{DISCUSSION}

ERCP is commonly employed to evaluate and treat pancreatic and biliary tract disease ${ }^{[1]}$. ERCP-related complications occur in up to $7 \%$ of patients, and mortality is estimated to be around $0.3 \%^{[1]}$. Septic complications are usually due to enteric bacteria ${ }^{[1]}$. Current guidelines suggest a benefit of pre-ERCP antibiotics only in those patients with known or suspected biliary obstruction in which complete drainage may not be achieved ${ }^{[1]}$.

C. perfringens is an anaerobic, gram-positive, rod-shaped bacterium that is part of the normal gastrointestinal microbiome ${ }^{[2]}$. It can become pathogenic if it gains access to the bloodstream. Normally, bacteria become bloodborne through mucosal damage ${ }^{[2]}$. This damage can result from intestinal neoplasia, chemotherapy-induced mucositis or instrumental or surgical procedures that disrupt the mucosal barrier (as seen in ERCP) ${ }^{[2]}$. There are only two case reports on PubMed describing C. perfringens infection after ERCP ${ }^{[1]}$.

Bacteriaemia due to $C$. perfringens is rare ( $1 \%$ of all positive blood cultures $)^{[3]}$ but is associated with fatal outcomes, especially when massive haemolysis occurs (associated with a mortality rate of $70-100 \%$ ) ${ }^{[3]}$, as seen in our case. Massive haemolysis in C. perfringens infection is triggered by bacterial toxins that can disrupt the erythrocyte cell membrane due to phospholipase $\mathrm{C}$ activity, causing morphological changes (spherocytes) and haemolysis ${ }^{[4]}$. As C. perfringens has a doubling time of only around 7 minutes, this bacterium can produce toxins very rapidly and contribute to the patient's rapid deterioration ${ }^{[4]}$. Due to the catastrophic clinical picture often encountered with these cases, a correct diagnosis can be difficult to obtain. When facing an icteric, febrile patient with abdominal pain, there must be a high index of suspicion for this infectious agent. Apart from clinical clues such as the presence of risk factors (old age, diabetes, immunosuppression, abdominal instrumentation) ${ }^{[5]}$, the laboratory test results are paramount in confirming $C$. perfringens infection. Specific clues include: acute haemoglobin reduction, with evidence of intravascular haemolysis (low haptoglobin levels, haemoglobinuria, haemolyzed blood samples), a peripheral blood smear with spherocytes but no schistocytes, and a negative Coombs test ${ }^{[4]}$. Other non-specific findings include those associated with bacterial sepsis (a granulocyte left shift, toxic granulation, Döhle bodies and cytoplasmatic vacuoles) and its complications (schistocytes if disseminated intravascular coagulation develops) ${ }^{[3]}$. A definite diagnosis depends on blood or tissue cultures. Treatment should be started as soon as possible as the median time from admission to death in these patients is 9.7 hours ${ }^{[5]}$. Surgical identification and removal or drainage of the focus of infection should carried out as it is associated with increasing survival ${ }^{[5]}$. Antibiotic therapy should be started as soon as possible and should include a penicillin or penicillin-derived antibiotic associated with clindamycin or metronidazole as the last two have activity against $C$ lostridium toxins, inhibiting their synthesis ${ }^{[5]}$. Massive transfusion may be necessary ${ }^{[5]}$. Some authors also propose hyperbaric oxygen therapy, but the evidence is not strong, as few patients have been submitted to this treatment ${ }^{[4]}$.

\section{REFERENCES}

1. Bari K, Aslanian HR, Pollak J, Reiner E, Salem RR, Taddei TH, et al. Emphysematous cholecystitis resulting in secondary biliary cirrhosis: a rare complication of endoscopic retrograde cholangiopancreatography. ACG Case Rep J 2013;1(1):51-54.

2. Smit B, van der Helm MW, Bosma M, Hudig F, Russcher H. Massive hemolysis due to Clostridium perfringens: a laboratory's perspective. Clin Chem Lab Med 2020;58(11):e295e297.

3. García Carretero R, Romero Brugera M, Vazquez-Gomez O, Rebollo-Aparicio N. Massive haemolysis, gas-forming liver abscess and sepsis due to Clostridium perfringens bacteraemia. BMJ Case Rep 2016;2016:bcr2016218014.

4. Hashiba M, Tomino A, Takenaka N, Hattori T, Kano H, Tsuda M, et al. Clostridium perfringens infection in a febrile patient with severe hemolytic anemia. Am J Case Rep 2016;17:219-223.

5. Simon TG, Bradley J, Jones A, Carino G. Massive intravascular hemolysis from Clostridium perfringens septicemia: a review. J Intensive Care Med 2014;29(6):327-333. 